\title{
Scroll wave instability controlled by external fluctuations
}

\author{
F. Sagués, ${ }^{a}$ S. Alonso, ${ }^{a}$ and J.M. Sancho ${ }^{b}$ \\ ${ }^{a}$ Departament de Química Física, Universitat de Barcelona, Diagonal 647, \\ E-08028 Barcelona, Spain \\ ${ }^{\mathrm{b}}$ Departament d'Estructura i Constituents de la Matèria, Universitat de \\ Barcelona, Diagonal 647, E-08028 Barcelona, Spain
}

\begin{abstract}
Scroll waves, a characteristic dynamical pattern of three dimensional excitable media, exhibit an instability under low excitability conditions. This unstable regime can be partially controlled by using random forcing in a clear manifestation of the ordering role of a stochastic external perturbation. Analytical and numerical results confirm this unexpected noise effect.
\end{abstract}

Key words:

Scroll waves, Excitable media, Order out of noise

PACS: 82.40.Bj, 05.40.-a, 47.54.+r

\section{Introduction}

Scroll waves and scroll rings are examples of dynamical patterns characteristic of three dimensional excitable media which appear, for example, in cardiac tissue or in the Belousov-Zhabotinsky chemical reaction. These waves are generated and rotate around a line defined by a phase-singularity, called the filament. In the case of scroll rings the filament forms a closed ring. Occasionally, these propagating waves degenerate into chaotic regimes. Such unstable regimes have been identified with the fibrillation state in ventricular tissue preceeding cardiac death [1]. Under low excitability conditions of the medium, actually near the subexcitable limit, the filament presents an unstable dynamical regime bending over itself, giving rise to a endless growing entangled coil [2] and finally leading to a highly disorganized pattern of wave propagation $[3]$.

Preprint submitted to Elsevier Science $\quad 27$ September 2004 
From a theoretical point of view this regime has been identified with a negative tension in the filament [4] thus implying its desestabilization. The rate of growth of the perturbations is inversely proportional to the local curvature $\left(\kappa^{-1}\right)[2]$; thus, these episodes of turbulence are associated with conditions of expansion of scroll rings. On the other hand, when this instability does not exist and the tension associated with the filament is positive, the scroll ring collapses.

Our objective in this work is to investigate the possibility of using a random forcing as a control technique to prevent such a turbulent-like state. In what follows we will prove that this objective can be indeed achieved in a new manifestation of the ordering role of a stochastic perturbation $[5,6]$.

From this perspective, theoretical investigations in model systems on how to control and avoid this regime may have future practical relevance. In this respect a very simple scenario such as Barkley's model has been used for what follows. In the next section, this model is presented with its corresponding phase diagram for the different regimes. In Section 3, numerical evidence is presented about the noise effects on this model. In Section 4 we prove analytically that the noise indeed produces ordering effects and finally we end with some conclusions and comments in Section 5.

\section{Modelization}

Excitable media [7] are ubiquous in many different fields such as physics, chemistry, biology, etc, and accordingly many diverse theoretical models have been proposed to describe specific scenarios: the Oregonator, Hodgkin-Huxley, Fitz-Hugh Nagumo, Barkley, and many more. Nevertheless all of then share the same phenomenology: they can sustain a rich variety of dynamical waves according to a well-defined characteristic of the medium: its excitability.

Here for the sake of simplicity we will use one of the simplest models from analytical and numerical tractability point of view: the so called Barkley's model [8]. It, under external noise forcing, reads,

$$
\begin{aligned}
& \partial_{t} u=D_{u} \nabla^{2} u+\frac{1}{\epsilon} u(1-u)\left(u-\frac{v+b+\xi(\mathbf{x}, t)}{a}\right) \\
& \partial_{t} v=u-v
\end{aligned}
$$

where we have introduced the noise through the parameter $b \rightarrow b+\xi(\mathbf{x}, t)$. This choice corresponds to the fact that this parameter is singularly relevant in determining the excitability of the medium. The stochastic process $\xi(\mathbf{x}, t)$ 


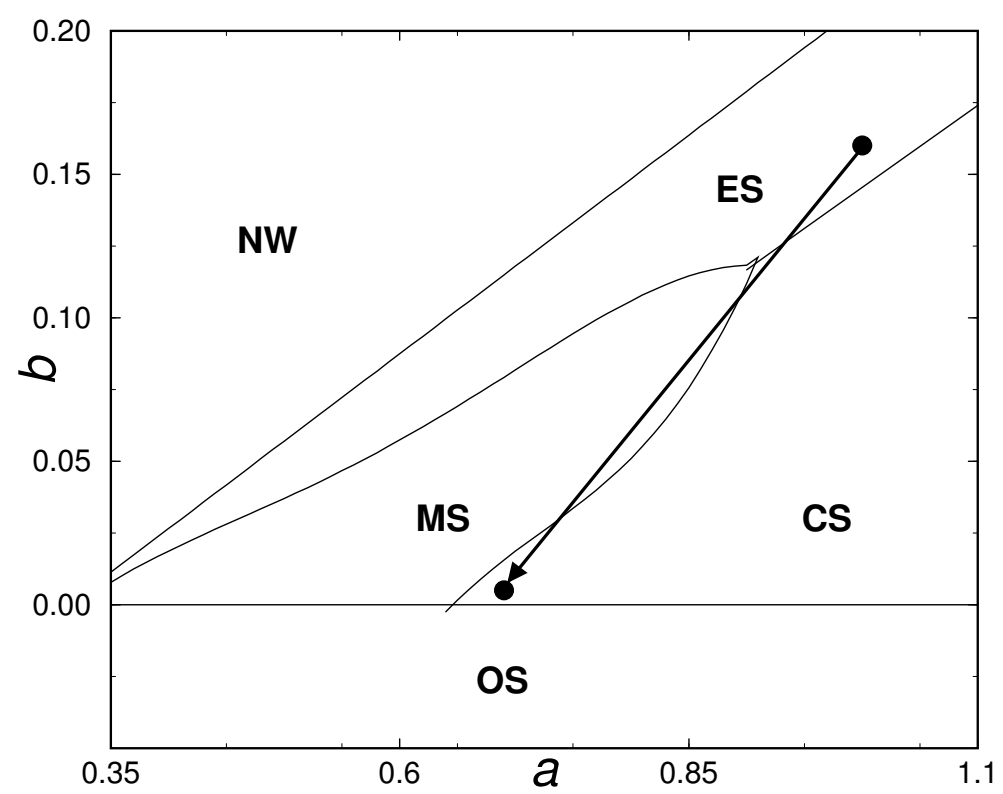

Fig. 1. Phase diagram of the dynamical regimes of the Barkley model. The parameter regions are NW (no waves), MS (meandering spirals), CS (collapsing scroll rings), ES (expanding scroll rings) and OS (oscillatory). The values of the other parameters are $\varepsilon=0.02$ and $D_{u}=1$. The line connecting the two points shows the change induced by noise (see text below and Fig. 5).

is assumed to be a gaussian white noise with zero mean and correlation [5],

$$
\left\langle\xi(\mathbf{x}, t) \xi\left(\mathbf{x}^{\prime}, t^{\prime}\right)\right\rangle=2 \sigma^{2} \delta\left(t-t^{\prime}\right) \delta\left(\mathbf{x}-\mathbf{x}^{\prime}\right)
$$

This assumption is made for simplicity, although the main noise effects do not depend qualitatively on the particular statistics chosen. [9].

The corresponding phase diagram of this model is plotted in Fig. 1, as a function of the most relevant system parameters $a$ and $b$ [10]. Of particular interest in our study are regions ES (expanding scrolls) and CS (collapsing scrolls). We will prove here that it is possible to move the system from ES to $\mathbf{C S}$ regimes by increasing the intensity of the external noise, inducing the elimination of the negative tension in the filament. Other changes are also possible and commented on the conclusions.

\section{Phenomenology}

Rotating scroll waves can be seen as a set of two-dimensional spirals stacked one upon another rotating around a filament (which connects the centers of the spirals). Thus, the free edge of the three-dimensional wave rotates around 

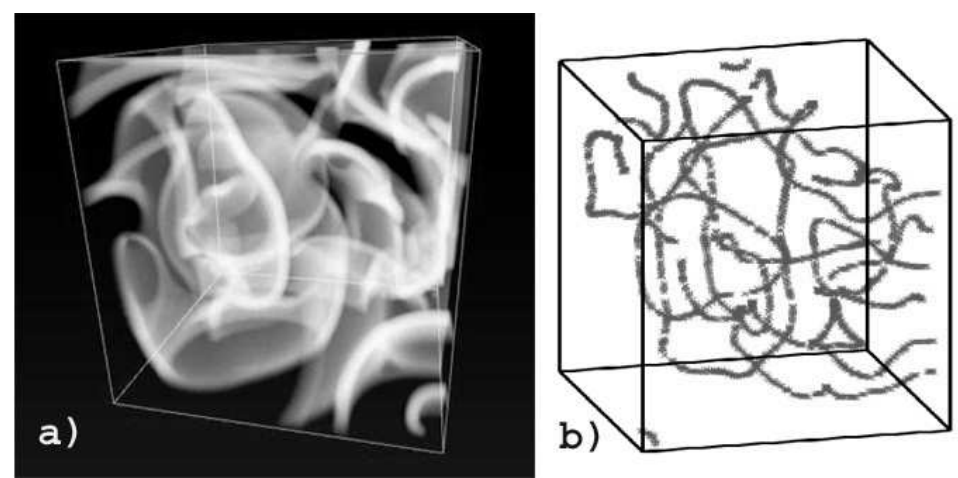

Fig. 2. (a) Disorganized propagation of waves in three-dimensional excitable media under conditions of weak excitability. (b) Snapshot of the filaments for the same realization.

this filament and the system can develop complex dynamics. In Fig. 2 we see a plot of a realization of such a scroll wave instability and its corresponding isolated filament from a different perspective. The dynamics of the scroll can be understood by analyzing the behavior of the filament.

Under conditions of weak excitability, the length associated with this filament increases sharply due to its negative linear tension. In Fig. 3.a the evolution of filament length is presented. It is clear how the instability develops in the first stages of the evolution. Moreover, the filament remains connected until it touches the boundaries where it breaks and then gives rise to the oscillations observed for large times in this figure.

In Fig 3.b the power spectra of the dynamics of a single point of the medium for a two-dimensional stable spiral wave and for the negative tension turbulent regime are compared. The first difference observed in the 3D turbulent system with respect to the $2 \mathrm{D}$ one is the loss of coherence of the dynamics due to the chaotic properties of this regime. However, some characteristic frequencies remain, not as sharp as in the $2 \mathrm{D}$ case, due to the rotation of the propagating waves around the unstable filament. Furthermore, it is interesting to remark that there is a displacement of the characteristic frequency to larger values for the unstable regime with respect to the rigid rotation of $2 \mathrm{D}$ spiral waves.

Another picture of this instability regime, showing more clearly how we can control it, is plotted in the spots of Fig. 4. The initial state is a straight filament with the two ends located at the upper and lower plane boundaries of a cubic slab. As time goes on the filament starts to display more and more complicated and incoherent patterns of wave propagation, giving rise to an increase of the length, as already discussed.

Nevertheless, in the same Fig. 4, we can appreciate the strong effects of an intermediate stochastic forcing once such a perturbation is fed into the system. Indeed, after the introduction of the noise, the filament starts to stretch out 

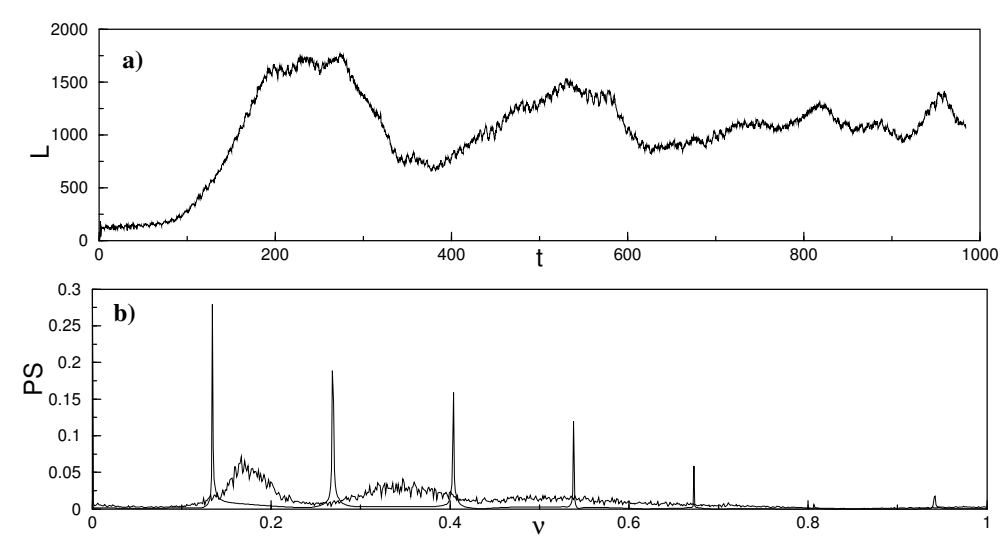

Fig. 3. (a) Time dependence of the total length of a circular filament for the parameter values, $a=1.1$ and $b=0.19$ (see Eq. (1)). (b) Power spectrum of the turbulence for the parameter values, $a=1.1$ and $b=0.21$; the thin lines show the power spectrum for a spiral wave in the $2 \mathrm{D}$ medium.

to adopt quite straight conformations with obviously random displacements. It turns out clearly that some amount of noise can eliminate this instability. However, for high enough noise intensities (not shown in the figure), the system develops a highly disorganized or chaotic pattern of wave propagation, as should be expected, because diverse nucleations of waves are generated by these perturbations.

\section{Theoretical analysis and results}

According to our previous results [9], the ordering role of the external noise in this system can be interpreted acting so as to enhance the excitability of the medium and accordingly to stabilize dynamical structures characteristic of this regime.

This fact can analytically be proved by analyzing the mathematical consequences of the random term in the equations of motion (1). As we are considering real systems the multiplicative white noise has to be interpreted in the Stratonovich sense. Accordingly, standard lowest order calculations in the noise intensity give a finite contribution for the averaged equation for the activator concentration. It reads,

$$
\langle u(1-u) \xi(\mathbf{x}, t)\rangle=\frac{\sigma^{2}}{\Delta x^{3}}\langle u(1-u)(1-2 u)\rangle
$$

Now, and due to the special analytical properties of the Barkley model, this contribution can be absorbed in a redefinition of the model parameters $\epsilon, a$ and $b$. In particular the changes induced in the last two parameters are of 


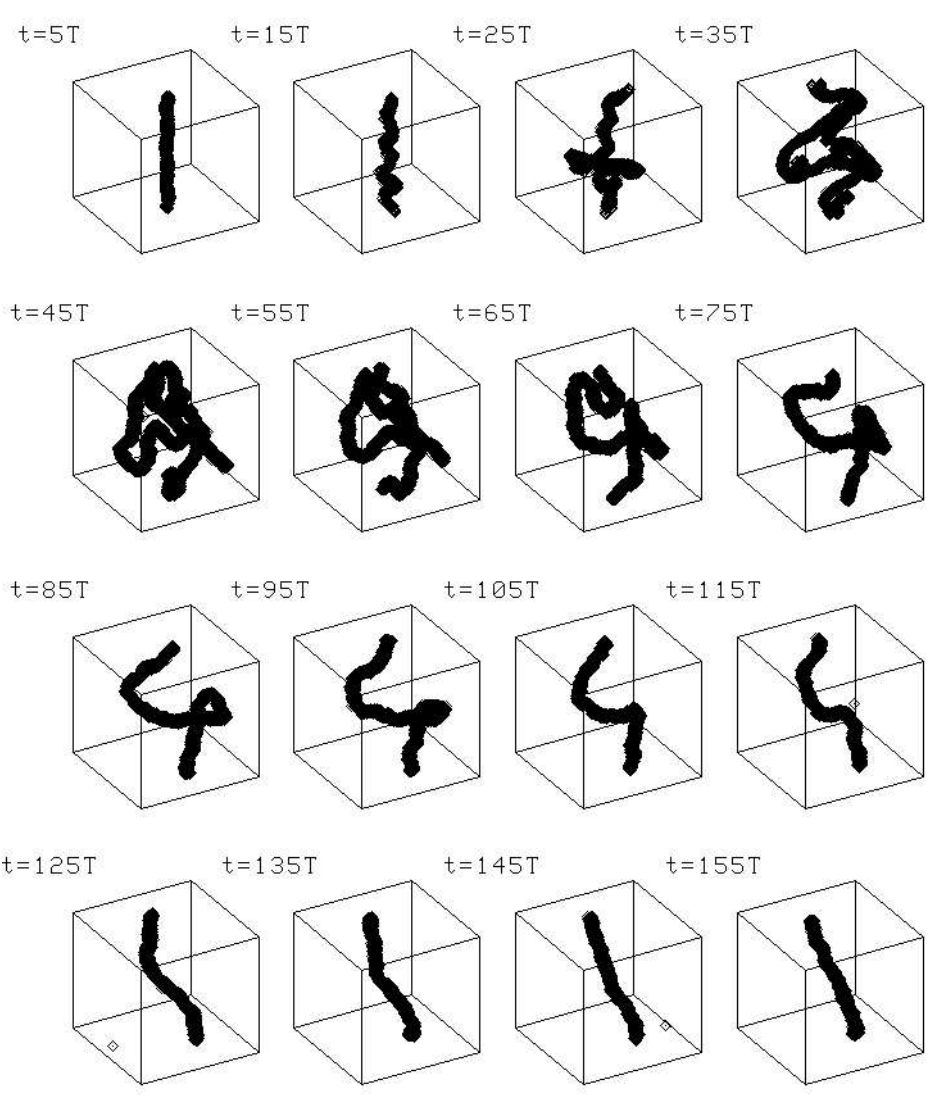

Fig. 4. Evolution of an initially unstable filament with the two ends fixed. At time $t=40 T$, a random forcing is added to the medium $\left(\sigma^{2}=0.0002\right)$. Points pertaining to the filament are calculated using the prescription $u_{f}=1 / 2$ and $\partial_{t} u_{f}=0$ during a whole period of rotation of the scroll wave around the vortex filament $(T=4.5)$. Model parameter values are: $a=1.0, b=0.16$. The simulation was run in a box of $150 \times 150 \times 150$ pixels with $\Delta x=0.4$ and $\Delta t=0.01$.

special relevance $[9]$,

$$
a^{\prime}=a\left(1-\frac{2 C(0)}{\epsilon a^{2}}\right) ; \quad b^{\prime}=b-\frac{C(0)}{\epsilon a}
$$

where $C(0)=\frac{\sigma^{2}}{\Delta x^{3}}$ is the local noise intensity in each lattice cell. These changes are plotted in Fig. 5. These new parameters drive the system to a different regime of larger excitability as it can be seen in Fig. 1, with lower effective values of the parameters $a$ and $b$ respectively. The variation of $\epsilon$ with the noise is small and it can be ignored as a first approximation. However, if the modification in $\epsilon$ is considered, the definition of a new set of parameters $a, b$ and $\epsilon$ is also possible, and the renormalized system may be solved numerically [11]. 

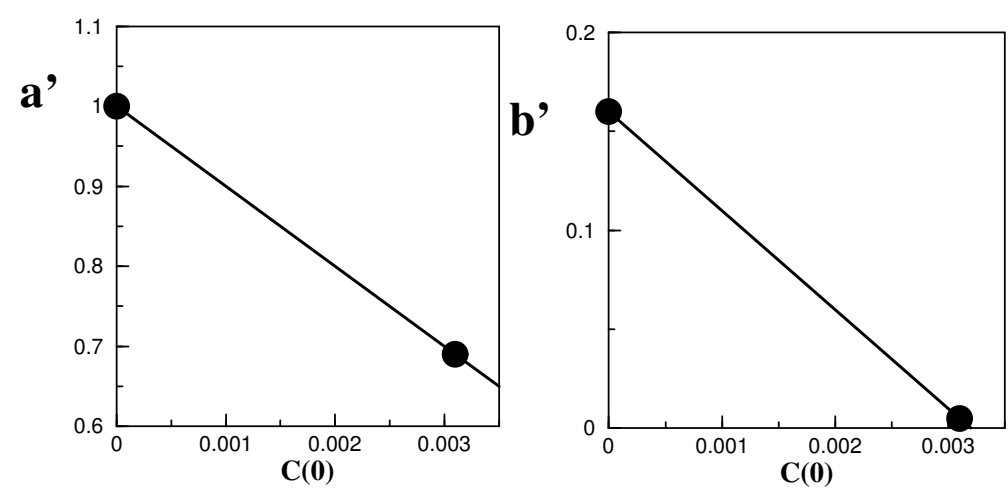

Fig. 5. Effective parameters, Eq.(4), of the Barkley model as a function of the noise intensity. Points show the deterministic initial unstable state and the noisy stable ones corresponding to Fig. 4.

\section{Conclusions}

An example of the counterintuitive ordering role of external noise superimposed on a complex dynamical 3D system has been presented. The instability of scroll waves in the low excitability regime can be tamed by feeding spatiotemporal stochastic fluctuations into one of the parameters which controls the excitability of the system. Similarly to this transition in 3D excitable media, more examples were obtained in two [12,13] or one dimensional systems [14]. Stochastic forcing is able to induce stability to non-excitable waves which deterministically decay and die [15] and to sub-excitable waves with free ends which initially contract and disappear [12], and even produces target waves under excitable conditions [13].

A last comment is in order. As we have seen in the last section, an increment of the noise intensity drives the system (see the phase diagram of Fig. 1) along the direction of the diagonal connecting the upper right corner to the left lower one. Accordingly we have more choices to change the dynamical regime such as, for example, from MS or CS to OS, etc., by the application of the same mechanism of increasing the noise intensity. These are also other examples of the ordering role of external fluctuations mentioned above.

Although the results in this text are mostly numerical, a theoretical analysis has been presented and interpreted as another example of noise-induced excitability transitions or, in general, as a striking manifestation of noise-induced order.

This research was supported by the Ministerio de Ciencia y Tecnología (Spain) and FEDER under projects BFM2003-07850, BQU2003-05042-C02-01 and 2001SGR00045. S. A. was financially supported by a grant from MCyT. 


\section{References}

[1] F. H. Fenton, E. M. Cherry, H. M. Hastings, S. J. Evans, Chaos 12 (2002) 852.

[2] S. Alonso, R. Kähler, A. S. Mikhailov, F. Sagués, accepted in PRE.

[3] A. T. Winfree, SIAM Rev. 32 (1990) 1.

[4] V. N. Biktashev, A. V. Holden, H. Zhang, Phil. Trans. Roy. Soc. A 347 (1994) 611.

[5] J. García-Ojalvo and J. M. Sancho, Noise in Spatially extended Systems (Springer-Verlag, New York, 1999).

[6] F. Sagués, J. M. Sancho, Patterns out of noise (To appear in Contemporary Physics, 2004)

[7] A. S. Mikhailov, Foundations of Synergetics I. Distributed Active Systems (Springer, Berlin, 1990)

[8] D. Barkley, M Kness, L. S. Tuckerman, Phys. Rev. A 42 (1990) 2489.

[9] S. Alonso, F. Sagués, J. M. Sancho, Phys. Rev. E 65 (2002) 066107.

[10] S. Alonso, F. Sagués, A. S. Mikhailov, Science 299 (2003) 1722.

[11] S. Alonso, F. Sagués, J. M. Sancho. Submitted PRE.

[12] S. Kádár, J. C. Wang, K. Showalter, Nature 391 (1998) 770.

[13] S. Alonso, I. Sendiña-Nadal, V. Pérez-Muñuzuri, J. M. Sancho, F. Sagués, Phys. Rev. lett. 87 (2001) 078302.

[14] R. Bascones, J. García-Ojalvo, J.M. Sancho, Phys. Rev. E 65 (2002) 061108.

[15] L. Q. Zhou, X. Jia, Q. Ouyang, Phys. Rev. Lett. 88 (2002) 138301. 